\title{
CINNAMON REDUCES DYSLIPIDEMIA AND LIVER STEATOSIS INDUCED BY HIGH FAT DIET IN ALBINO RATS: HISTOLOGICAL, ULTRASTRUCTURAL, AND BIOCHEMICAL STUDIES
}

\author{
Samah M. Arisha*; Saber A. Saker; Fatma R. Abd El-Haseeb \\ Zoology Department, Faculty of Science, Menoufia University, Menoufia, Egypt
}

\author{
Article History: \\ Received: 09 May 2020 \\ Revised: 30 May 2020 \\ Accepted: 13 June 2020 \\ Published Online: \\ 17 June 2020 \\ Keywords: \\ Cinnamon \\ Hyperlipidemia \\ Liver pathology \\ Liver ultrastructure \\ Steatosis \\ *Correspondence: \\ Samah Arisha \\ Zoology Department \\ Faculty of Science \\ Menoufia University \\ Menoufia, Egypt \\ E-mail: \\ arishasamah@yahoo.com
}

\begin{abstract}
High fat diet (HFD) induces hyperlipidemia and causes non-alcoholic fatty liver disease. Cinnamon (Cinnamomum zeylanicum) has many curative effects as anti-inflammatory and antioxidant. This study aimed to investigate the potential protective effect of cinnamon against HFD-induced dyslipidemia and liver steatosis. Forty adult male Wistar albino rats were used in the current study; these were allotted randomly to 4 equal groups. The animals were fed on standard diet (group 1), standard diet containing cinnamon powder " $15 \%$ weight by weight, w/w" (group 2), HFD (group 3), or HFD containing cinnamon powder " $15 \% \mathrm{w} / \mathrm{w}$ " (group 4 ) daily for 4 or 8 weeks. The results showed that HFD increased significantly $(P \leq 0.05)$ the body weight and the activities of serum aminotransferases, and induced dyslipidemia. Examination of the liver of the HFD-fed rats showed many pathological changes, especially intracytoplasmic fat accumulation either as small lipid droplets with variable sizes (microsteatosis) or large lipid droplets (macrostatosis) that may displace the nucleus laterally. Ultrastructural examination of the same group showed various degenerative alterations in the nucleus, mitochondria, endoplasmic reticulum, and Kupffer cells, as well as lipid accumulation. However, examination of liver of animals fed on HFD+cinnamon showed a remarkable improvement especially after 8 weeks; the histological and ultrastructure pictures were mostly similar to the control group. In addition, the animals fed on HFD+cinnamon recorded a decrease in the body weight and improvement in readings of the biochemical parameters compared with the HFD-treated group. In conclusion, cinnamon alleviated the occurrence of non-alcoholic fatty liver disease in the HFD-fed rats.
\end{abstract}

\section{INTRODUCTION}

Dyslipidemia is a metabolic disorder disease associated with an excess in blood total cholesterol (TC), triacylglycerol (TG), and low density lipoprotein-cholesterol (LDL-C) levels, as well as a decrease in the high density lipoprotein-cholesterol (HDL-C) level $^{[1]}$. It causes non-alcoholic fatty liver disease (NAFLD) ${ }^{[2]}$. High fat diets (HFD) with or without cholesterol contribute to favor hepatic lipid storage in microvesicular and macrovesicular steatosis and 
the induction of inflammation, which plays a pivotal role in conjunction with obesity to cause shortage in antioxidant defense capacity with an increase in the oxidative stress due to the overproduction of reactive oxygen species $(\mathrm{ROS})^{[2,3]}$. The accumulation of cholesterol rather than TG may play a critical role in the progression from simple steatosis to steatohepatitis ${ }^{[4]}$. Because the synthetic chemical-drugs prescribed for treating obesity have many adverse side effects, therefore there is a great need to search for alternative natural agents from medical plants, herbs, and spices. Cinnamon is an important age-old spice and aromatic crop having wide applications as flavoring, perfumery, and medicine agent. It is used for many years in traditional medicine; since it had many health benefits such as anti-inflammatory, antioxidant, anticancer, astringent, anticlotting, antihyperlipidaemic, antidiabetic, and antimicrobial properties $^{[5-9]}$. The cinnamon contains a wide range of essential oils, such as transcinnamaldehyde, cinnamyl acetate, eugenol, L-borneol, caryophyllene oxide, $\beta$-caryophyllene, L-bornyl acetate, E-nerolidol, $\alpha$ cubebene, $\alpha$-terpineol, terpinolene, $\alpha$-thujene, cinnamic acid, cinnamate, cinnamaldehyde, and other components as polyphenols ${ }^{[10,11]}$. Tuzcu et $a l .{ }^{[12]}$ concluded that cinnamon polyphenols reduces the hyperlipidemia, inflammation, and oxidative stress through activating transcription factors and antioxidative defense signaling pathway in rat liver fed on high HFD. The most abundant anti-inflammatory compounds in cinnamon are E-cinnamaldehyde and $O$ methoxycinnamaldehyde ${ }^{[13]}$. Askari et al ${ }^{[14]}$ suggested that cinnamon may be effective in improving blood lipid profile and insulin sensitivity in patients with non-alcoholic fatty liver disease. Most of these studies relied on the changes in the biochemical parameters or the histological architecture without investigating the changes in the liver ultrastructure. Therefore, this study aimed to evaluate the potential protective effect of cinnamon against the dyslipidemia and liver steatosis induced by HFD, with especial reference to liver histopathological and ultrastructural changes.

\section{MATERIAL AND METHODS \\ Materials}

Cinnamon (Cinnamomum zeylanicum), family Lauraccae, was purchased from local market of agricultural herbs and medical plants (Cairo, Egypt), and authenticated by a specialist taxonomist at the Botany Department, Faculty of Science, Menoufia University. The cinnamon bark was grinded using electric mixer into a fine powder then added to animal's diet $(15 \%$ weight by weight, $w / w)^{[15]}$. A standard animal diet manufactured especially for laboratory purposes was obtained from the Egyptian Company of Oils and Soap (Kafr El-Zayat, Gharbia, Egypt). Moreover, materials used for preparing the HFD (cholesterol, sucrose, cellulose, etc.) were purchased, as a pure powder, from El-Gomhouria Co. (Cairo, Egypt). Table "1" showed the formula of the standard diet and HFD used in these experiments.

Table (1): The formula of the standard diet and high fat diet.

\begin{tabular}{lcc}
\hline Contents & $\begin{array}{c}\text { Standard diet } \\
(100 \mathrm{~g})\end{array}$ & $\begin{array}{c}\text { High fat diet } \\
(100 \mathrm{~g})\end{array}$ \\
\hline Casien & 10 & 10 \\
Cellulose & 4.7 & 4.7 \\
Cholesterol & - & 2 \\
Cocca butter & - & 15 \\
Corn starch & 5 & 15 \\
Sucrose & - & 15 \\
Wheat & 76 & 34 \\
Mineral mixture & 3.3 & 3.3 \\
Vitamin mixture & 1 & 1 \\
\hline
\end{tabular}

\section{Animals and study design}

Forty adult male Wistar albino rats (Rattus norvegicus), approximately two months old (weighting between 120-130g), were obtained from Egyptian Company Nile for pharmaceuticals and chemical 
industries (Cairo, Egypt). The animals were acclimatized for about ten days to the laboratory environment before the initiation of the experiment, and fed on a standard pellet diet and water ad libitum. Animals were maintained in plastic cages under restrained temperature $\left(23 \pm 2^{\circ} \mathrm{C}\right)$ and 12 hours light/dark. The protocol of this study was approved by the Ethics Committee of Faculty of Science, Menoufia University (Approval No. MUFS/F/HI/5/20). Experimental animals were randomly divided into 4 groups (10 rats in each group): group 1 (control group), fed on a standard diet; group 2 (cinnamon group), fed on a standard diet containing cinnamon powder (15\% w/w); group 3 (HFD group), fed on HFD to induce acute hyperlipidemia; group 4 (HFD+cinnamon group), fed on HFD containing cinnamon powder $(15 \% \mathrm{w} / \mathrm{w})$ daily for 4 or 8 weeks.

\section{Blood sampling:}

At the end of experiments (4 or 8 weeks), the rats were fasted overnight before killing. To achieve animal sedation, thick Gamgee with a hole of center was put on the face of animal and few drops of chloroform added only through the first minute. The blood samples were withdrawn from the cardiac puncture. Blood was allowed to coagulate at room temperature for 30 minutes to obtain serum then centrifuged at $4000 \mathrm{rpm}$ for 15 minutes. The separating serum was kept frozen at $-20^{\circ} \mathrm{C}$ until used for the biochemical analysis. Liver tissue was immediately removed out and prepared for the histological and ultrastructure studies.

Histological/ultrastructural examination:

Some pieces of liver were immediately fixed in $10 \%$ neutral formalin. After fixation liver sampled for histological examination were dehydrated and embedded according to the standard sampling trimming procedures. Sections of 4-5 $\mu \mathrm{m}$ thick were prepared and stained with the hematoxylin and eosin.

For the transmission electron microscopic examination, small liver specimens $(1.0 \mathrm{~mm})$ were fixed in $4 \%$ gluteraldehyde solution for 24 hours. Then they were post-fixed in $1.0 \%$ osmium tetroxide phosphate buffer $(\mathrm{pH}=7.4)$ for 2 hours, dehydrated, and embedded in Epon 812. Semi-thin sections were stained with $1.0 \%$ toluidine blue. Ultrathin sections were cut and stained with uranyl acetate and lead citrate. Chemicals used in histological and ultrastructure analyses were purchased from SigmaAldrich Corp (St. Louis, MO USA). The ultra-thin sections were examined with JEOL JEM-1400 plus transmission electron microscope (JEOL Ltd., Tokyo, Japan) in electron microscope unite of Faculty of Science, Alexandria University (Alexandria, Egypt).

\section{Biochemical studies}

The activities of aminotransferases (ALT and AST), as well as TC, TG, HDL-C, and LDL-C levels were estimated colorimetrically using commercial kits (Randox Laboratories Ltd., County Antrim, UK) according to the manufacturer's recommendation.

\section{Statistical analysis:}

The data are expressed as the mean \pm standard deviation. The student's $t$ test was used for the statistical analysis of the data. Differences were considered significant at $P \leq 0.05$.

\section{RESULTS}

Effect of cinnamon on the body weight and biochemical parameters in blood of obese rats

The standard diet containing $15 \%$ cinnamon did not significantly affect $(P>0.05)$ the body weight of animals (at the $4^{\text {th }}$ and $8^{\text {th }}$ weeks) as compared with the control group (Table 2). Whereas, a significant increase $(P \leq 0.05)$ in the body weight of HFD-fed animals was recorded after both 4 and 8 weeks of the treatment, as compared with the control group. On the other hand, animals received HFD+cinnamon $(15 \% \mathrm{w} / \mathrm{w})$ showed a significant decrease in the body weight (at the $4^{\text {th }}$ and $8^{\text {th }}$ weeks) as compared with the HFD-fed animals (Table 2). 
Table 2: Effect of cinnamon treatment on the body weight, serum aminotransferases activities, and serum lipid profile of the high fat diet (HFD) feeding animals.

\begin{tabular}{|c|c|c|c|c|}
\hline & Control & Cinnamon & HFD & HFD+Cinnamon \\
\hline \multicolumn{5}{|l|}{ Body weight (g) } \\
\hline Day (0) & $112.60 \pm 3.50$ & $110.25 \pm 3.70$ & $114.87 \pm 4.52$ & $114.24 \pm 4.49$ \\
\hline $4^{\text {th }}$ Week & $147.60 \pm 5.60$ & $145.8 \pm 3.77$ & $199.21 \pm 6.83 *$ & $176.26 \pm 8.96^{\dagger}$ \\
\hline $8^{\text {th }}$ Week & $191.43 \pm 3.58$ & $182.6 \pm 3.65$ & $360.23 \pm 43.14 *$ & $252.22 \pm 252.20^{\dagger}$ \\
\hline \multicolumn{4}{|l|}{ AST activity (IU/L) } & $185 \pm 6.50^{\dagger}$ \\
\hline $8^{\text {th }}$ Week & $85.93 \pm 4.44$ & $75.92 \pm 2.03$ & $341.01 \pm 6.12^{*}$ & $127.21 \pm 5.72^{\dagger}$ \\
\hline \multicolumn{5}{|l|}{ ALT activity (IU/L) } \\
\hline $4^{\text {th }}$ Week & $89.7 \pm 3.16$ & $79.33 \pm 6.90^{*}$ & $171.20 \pm 7.59 *$ & $127.81 \pm 5.63^{\dagger}$ \\
\hline $8^{\text {th }}$ Week & $102.01 \pm 5.59$ & $93.34 \pm 5.40^{*}$ & $254.86 \pm 21.17 *$ & $110.44 \pm 5.72^{\dagger}$ \\
\hline \multicolumn{5}{|l|}{$\mathrm{TC}(\mathrm{mg} / \mathrm{dL})$} \\
\hline $4^{\text {th }}$ Week & $75.96 \pm 24.15$ & $73.03 \pm 1.87$ & $182.80 \pm 10.90 *$ & $132.60 \pm 10.76^{\dagger}$ \\
\hline $8^{\text {th }}$ Week & $103.80 \pm 3.49$ & $66.74 \pm 2.91 *$ & $240.75 \pm 18.40^{*}$ & $110.43 \pm 3.36^{\dagger}$ \\
\hline \multicolumn{5}{|l|}{$\mathrm{TG}(\mathrm{mg} / \mathrm{dL})$} \\
\hline $4^{\text {th }}$ Week & $77.20 \pm 4.43$ & $60.48 \pm 3.96$ & $147.40 \pm 6.31 *$ & $124.41 \pm 2.09^{\dagger}$ \\
\hline $8^{\text {th }}$ Week & $76.01 \pm 1.79$ & $64.92 \pm 1.65^{*}$ & $40.34 \pm 3.04^{*}$ & $108.01 \pm 2.57^{\dagger}$ \\
\hline \multicolumn{5}{|l|}{ HDL-C (mg/dL) } \\
\hline $4^{\text {th }}$ Week & $46.10 \pm 2.96$ & $62.93 \pm 4.47 *$ & $32.22 \pm 2.56^{*}$ & $40.82 \pm 1.75^{\dagger}$ \\
\hline $8^{\text {th }}$ Week & $44.7 .80 \pm 2.88$ & $49.30 \pm 5.15$ & $22.93 \pm 3.11 *$ & $44.14 \pm 1.27^{\dagger}$ \\
\hline \multicolumn{5}{|l|}{ LDL-C (mg/dL) } \\
\hline $4^{\text {th }}$ Week & $37.10 \pm 5.31$ & $29.38 \pm 1.60$ & $118.60 \pm 2.53^{*}$ & $98.56 \pm 6.86^{\dagger}$ \\
\hline $8^{\text {th }}$ Week & $38.53 \pm 2.17$ & $32.42 \pm 3.69$ & $164.73 \pm 8.59 *$ & $84.22 \pm 84.20^{\dagger}$ \\
\hline
\end{tabular}

Data are presented as the mean \pm stander deviation $(n=5)$. ALT: alanine aminotransferase, AST: aspartate aminotransferase, TC: total cholesterol, TG: triacylglycerol, HDL-C: high density lipoprotein-cholesterol, LDL-C: low density lipoprotein-cholesterol. $* P \leq 0.05$, as compared with the control group; ${ }^{\dagger} P \leq 0.05$, as compared with the HFD group.

The rats received the standard diet containing $15 \%$ cinnamon showed a significant decrease in the serum ALT activity (at the $4^{\text {th }}$ and $8^{\text {th }}$ weeks) and the levels of serum TC and TG (at the $8^{\text {th }}$ week only), while the serum HDL-C level increased significantly (at the $4^{\text {th }}$ week only), as compared with the control rats (Table 2). The HFD-fed animals showed a significant increase in all measured biochemical parameters in the serum after both 4 and 8 weeks of the treatment, except the TG (at the $8^{\text {th }}$ week) and HDL-C (at both $4^{\text {th }}$ and $8^{\text {th }}$ weeks) concentrations that decreased significantly, as compared with the control animals. On the other hand, when cinnamon added to HFD; the aminotransferases activities and the levels of TC, TG (at the $4^{\text {th }}$ week only), and LDL-C decreased significantly, while the TG (at the $8^{\text {th }}$ week only) and HDL-C levels increased significantly, in animals' sera after both 4 and 8 weeks of the treatment as compared with the HFD-fed group (Table 2).

\section{Cinnamon alleviated the histological} alterations in the liver of obese rats

The examination of specimens of liver of the control and cinnamon groups showed normal histological architecture of classic hepatic lobules and sinusoids, lined by endothelium and some von Kupffer cells. The hepatocytes appeared polyhedral with acidophilic cytoplasm contained centrally 
pale-stained nuclei (Figure 1). Examination of liver sections of animals treated with HFD for 4 weeks revealed multiple inflammatory areas scattered all over the tissue; in addition to vacuolated hepatocytes, pyknotic nuclei, mononuclear inflammatory cell infiltration around dilated veins, and proliferated bile duct were also observed (Figures 2a-c). Large number of hepatocytes contained small fat droplets that described condition known as microvesicular steatosis was also seen in the liver sections of animals treated with HFD for 4 weeks. All pathological features become more pronounced in liver sections obtained from animals treated with HFD for 8 weeks (Figure 2d); a marked fatty degeneration was prominent and ballooning hepatocytes with rarified cytoplasm and single large fat vacuole displaced the nuclei peripherally (macrovesicular steatosis).

Histological examination of liver sections of rats fed on HFD+cinnamon for 4 weeks exhibited less prominent histopathological changes; few scattered hepatocytes containing minute vacuoles or pyknotic nuclei (Figure 3a). The HFD+cinnamon-fed rats showed markedly alleviation of the hepatic lesions, where the central veins retained to the normal size, and both the hepatocytes and the blood sinusoids appeared almost similar to those of the control group (Figures 3a and b).
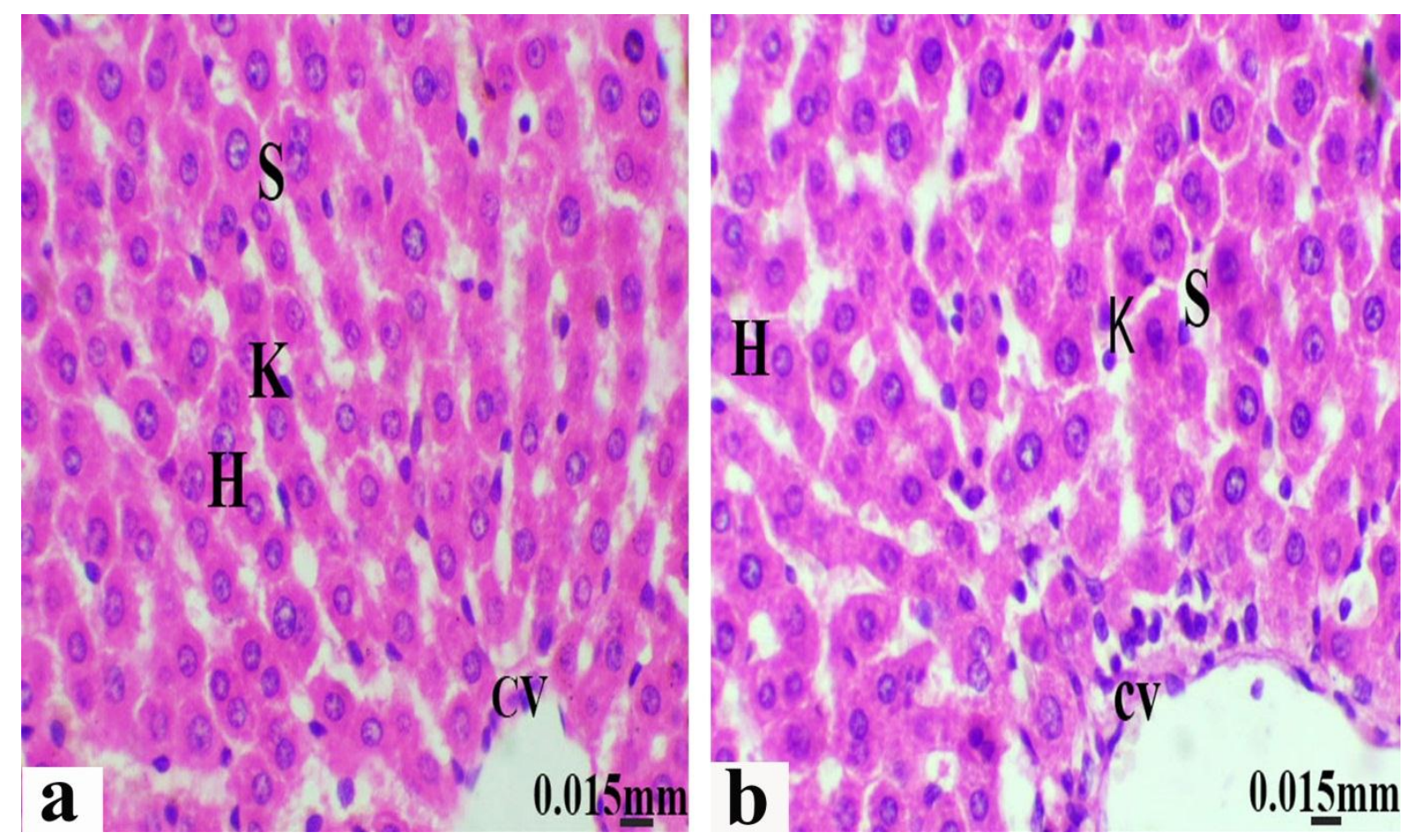

Figure 1: Photomicrograph obtained from liver section of: (a) Control rat showing central vein $(\mathrm{CV})$, radiating cords of hepatocytes $(\mathrm{H})$, sinusoids $(\mathrm{S})$, and Kupffer cells $(\mathrm{K})$. (b) Cinnamon-treated rat (at 8 weeks) showing normal hepatocytes $(\mathrm{H})$, sinosoids $(\mathrm{S})$, central vein $(\mathrm{CV})$, and Kupffer cells (K).

\section{Cinnamon alleviated the hepatic ultra- structure alterations of obese rats}

Electron microscopic examination of the liver sections of the control and cinnamon groups revealed normal ultrastructure of the hepatocytes (Figure 4). Their hepatocytes appeared with normal nucleus, mitochondria scattered within cytoplasm with well-developed cristae, and normal rough endoplasmic reticulum. In addition, the Kupffer cells appeared with normal nucleus and the bile canaliculi with short microvilli (projected to the lumen) are present between hepatocytes of the control and cinnamon groups (Figure 4). 

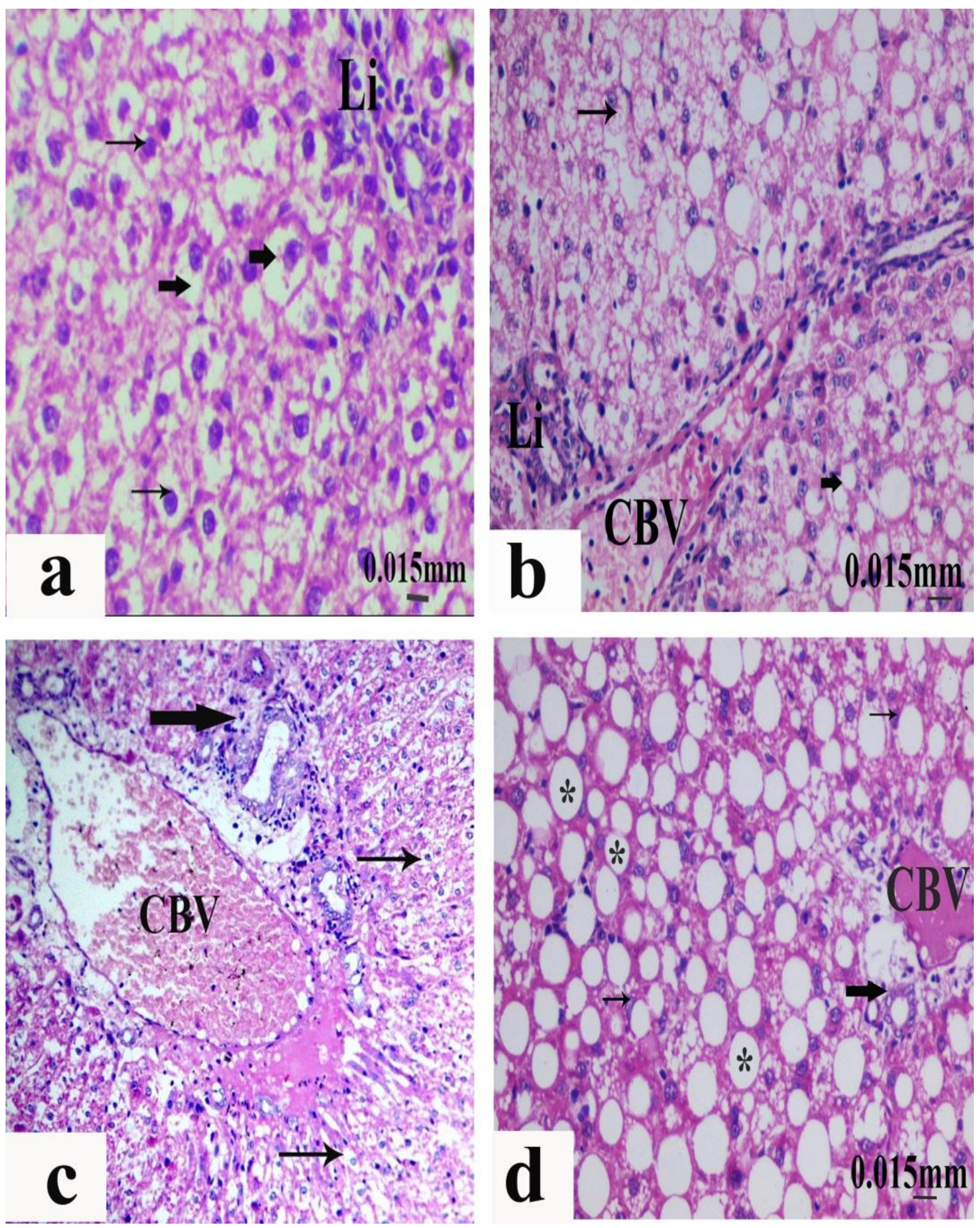

Figure 2: Photomicrograph obtained from liver sections of HFD-fed rat at 4 weeks showing (a) pyknotic nuclei (thin arrows), vacuolated hepatocytes (thick arrow), and leukocytic infiltration (Li); (b) microvesicular steatosis, hepatocytes with multiple small vacuoles (thin arrow), large coalesced vacuoles (thick arrow), congested blood vessel (CBV), and leukocytic infiltration (Li); (c) degenerated hepatocytes (thin arrows), congested blood vessel (CBV) and proliferation of bile ductules (thick arrow). (d) Liver section of HFD-fed rat at 8 weeks showing swollen hepatocytes with large fat vacuoles (*), peripheral nuclei (thin arrows), congested blood vessel (CBV), and bile duct (thick arrow). 

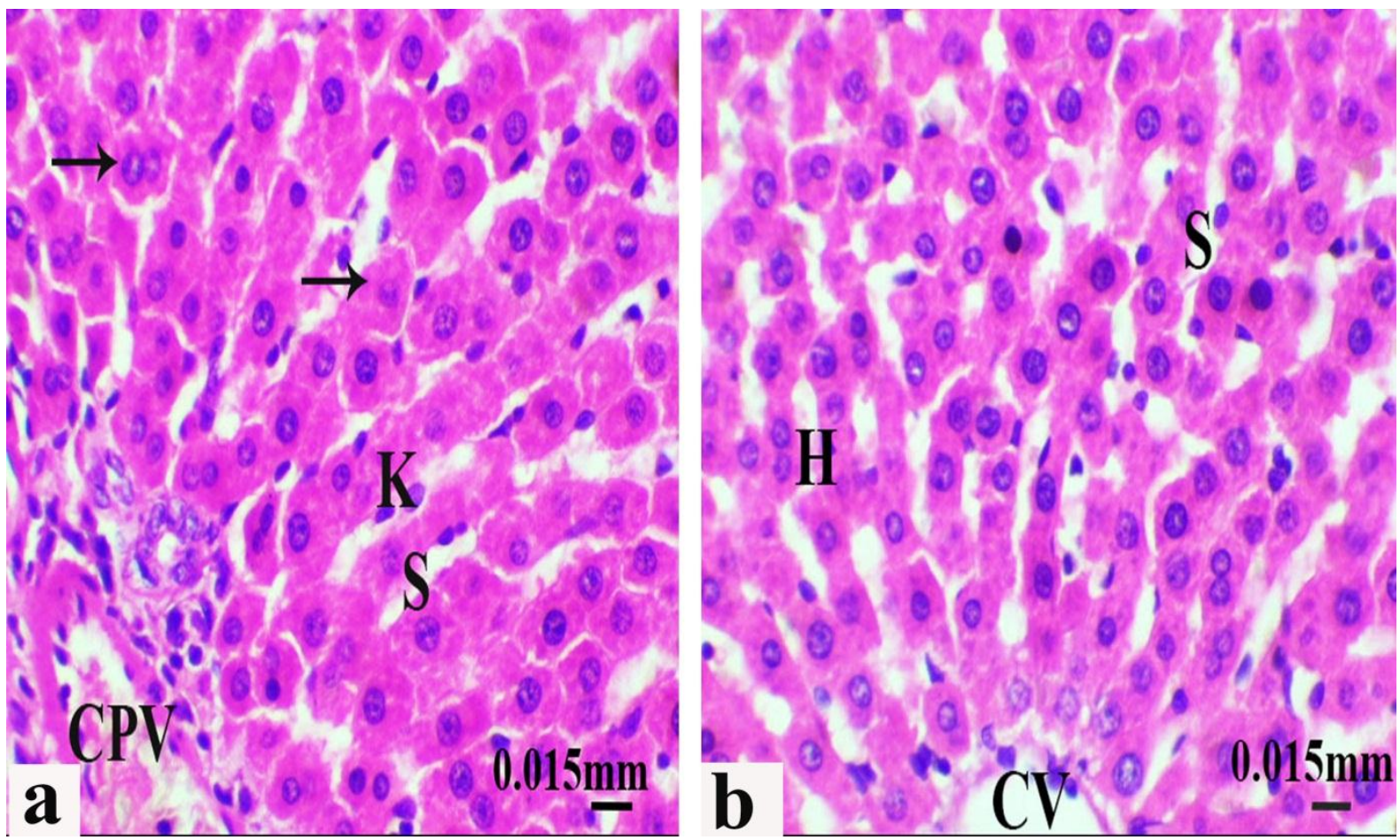

Figure 3: Photomicrograph obtained from liver section of: (a) Rat received HFD+cinnamon for 4 weeks showing improvement of architecture of hepatic lobules, normal hepatocytes (arrows), dilated sinusoids (S), mild congested portal vein (CPV), and few number of activated Kupffer cells (K). (b) Rat received HFD and cinnamon for 8 weeks showing central vein $(\mathrm{CV})$, nearly normal architecture of hepatic strands, hepatocytes $(\mathrm{H})$ and blood sinusoids $(\mathrm{S})$.

Animals fed on HFD for 4 weeks showed several ultrastructural changes including abnormal nuclei, irregular nuclear envelopes, rarified cytoplasm, dilated rough endoplasmic reticulum, degenerated mitochondria with complete or partial loss of their cristae, many lipid droplets with variable size (steatosis), and Kupffer cells with area of vacant cytoplasm (Figures 5a-c). The dramatic ultrastructural changes were observed in the hepatocytes of rats consumed HFD for 8 weeks; the cytoplasm contained large number of different sized lipid vacuoles, marginalized nuclei, with clumped chromatin, and degenerated cytoplasmic organelles (Figure 5d), as well as the degenerated Kupffer cells appeared in the dilated sinusoidal space (Figure 5e).

Examination of ultra-thin sections of liver obtained from animals fed on HFD+cinnamon for 4 weeks showed less prominent ultrastructural alternation; some abnormalities still observed as dilated rough endoplasmic reticulum, small lipid droplets and few lysosomes (Figure 6a). The ultrastructural findings of hepatocytes of rat received HFD+cinnamon for 8 weeks revealed an advanced degree of improvement; the nuclei, mitochondria, and rough endoplasmic reticulum seemed to be normal (Figure 6b), and bile canaliculi with normal organized microvilli were clearly seen (Figure 6c).

\section{DISCUSSION}

The present study recorded a significant elevation in the weight of the HFD-fed animals comparing with the control group that may be due to the deposition of fats in various body fat pads. This finding is consistent with the results obtained by Xia et $a .^{[16]}$ in the HFD-fed diabetic rat. Additionally, Fidèle et al. ${ }^{[17]}$ found that the cholesterol accumulation caused an increase 

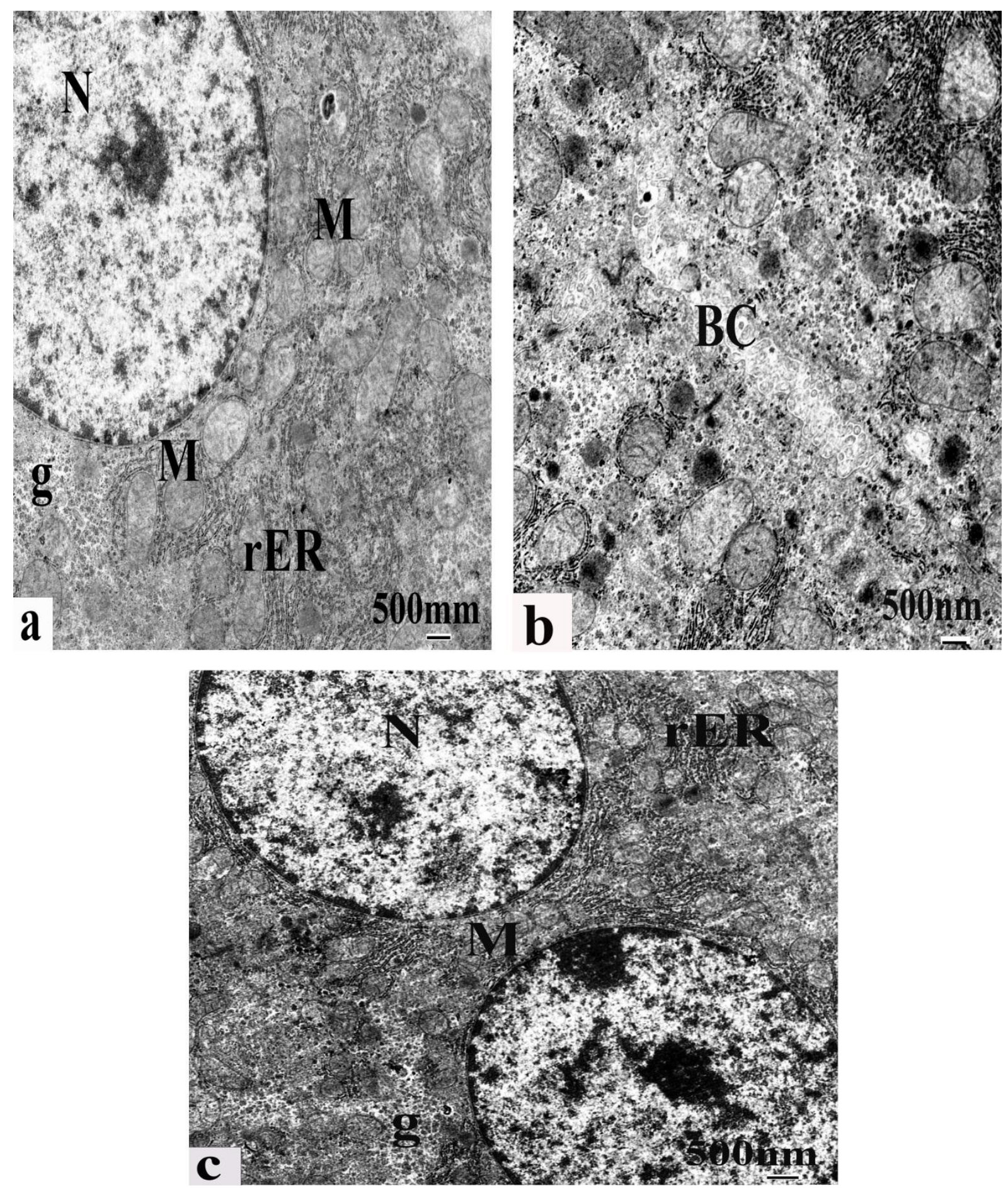

Figure 4: Electron micrograph of control animal showing (a) hepatocyte with normal nucleus $(\mathrm{N})$, regular nuclear envelope, normal mitochondria $(\mathrm{M})$, rough endoplasmic reticulum (rER) and glycogen rosettes (g); (b) bile canaliculi (BC). (c) Electron micrograph of an animal treated with cinnamon showing a hepatocyte with normal nucleus $(\mathrm{N})$, mitochondria $(\mathrm{M})$, rough endoplasmic reticulum (rER), and glycogen rosettes $(\mathrm{g})$.

in the rate of steroid hormone production such as cortisol, estrogens, and testosterone leading to an increase in the body weight of HFD-fed rats. Moreover, excessive growth of adipose tissue results in obesity includes two growth mechanisms: hyperplastic (an increase in cell number) and hypertrophic (an increase in cell size $)^{[18]}$. 

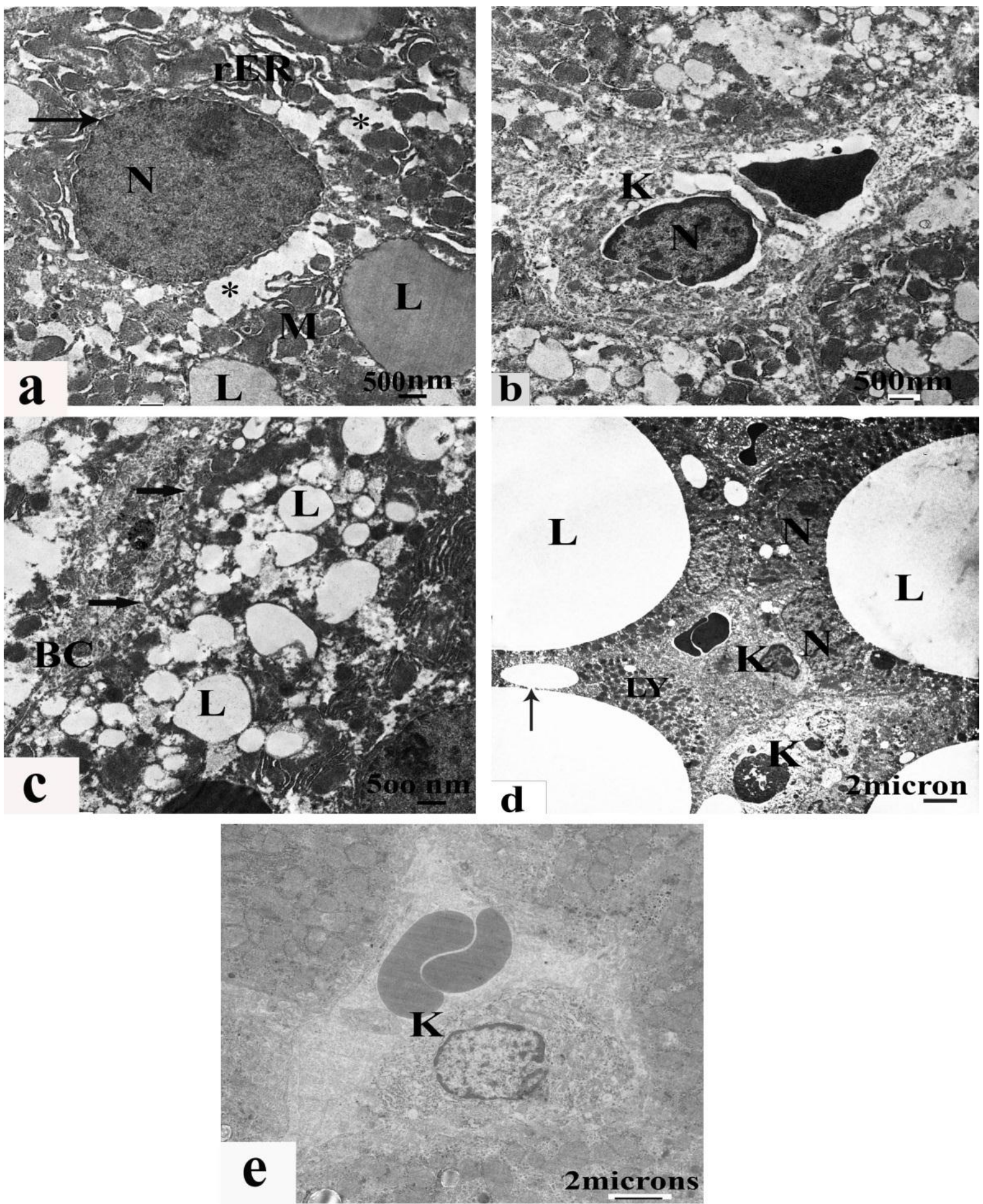

Figure 5: Electron micrograph of a hepatocyte of HFD-fed rat for 4 weeks showing (a) abnormal nucleus (N), irregular nuclear envelope (arrow), degenerated mitochondria (M), dilated rough endoplasmic reticulum (rER), lytic cytoplasm (*), and lipid droplets (L); (b) degenerated hepatocyte, sinusoidal space, Kupffer cell (K) with an area of vacant cytoplasm and normal nucleus (N); (c) bile canaliculus (BC) with cut-sections of microvilli (arrows) and part of degenerated hepatocyte with multiple lipid droplets (L). Electron micrograph of a hepatocyte of HFD-fed rat for 8 weeks showing (d) large lipid vacuoles (L), area of lipid fusion (arrow), compressed nuclei, Kupffer cell (K), and numerous lysosomes (LY); (e) degenerated Kupffer cell (K). 

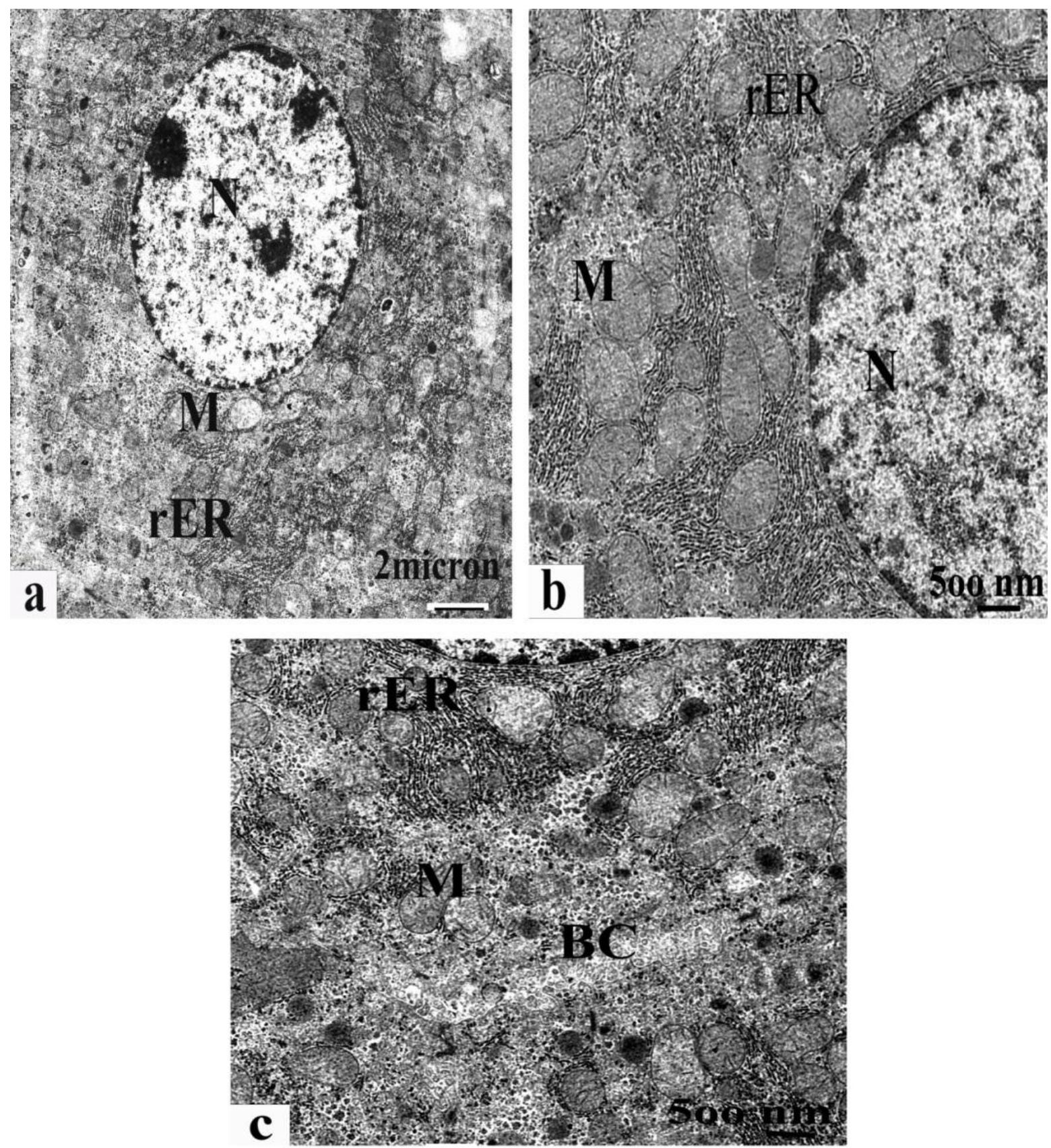

Figure 6: Electron micrograph of a part of a hepatocyte of an animal treated with: (a) HFD+cinnamon for 4 weeks showing normal nucleus $(\mathrm{N})$ with regular nuclear envelope, mitochondria (M) still have different degree of degeneration, and rough endoplasmic reticulum (rER); (b) HFD+cinnamon for 8 weeks showing normal nucleus (N) regular nuclear envelope, mitochondria $(\mathrm{M})$, and rough endoplasmic reticulum (rER); (c) HFD+cinnamon for 8 weeks showing normal bile canaliculi (BC) with regular microvilli and normal mitochondria $(\mathrm{M})$ with regular cristae.

In the present study, several histopathological changes were seen in the liver of HFD-fed animals either for 4 or 8 weeks; the hepatocytes undergo hydropic degeneration and become swollen and vacuolated, described as ballooning degeneration. The previous studies reported that the consumption of HFD may play crucial role in the pathogenesis of fatty liver resulting in hepatocellular damage and exaggerated hepatic steatosis that is associated with other factors such as 
oxidant stress, mitochondrial injury, fatty acids lipotoxicity, and inflammatory cytokines $^{[19,20]}$ Tveden-Nyborg et al. ${ }^{[21]}$ attributed these changes to the oxidative stress of dyslipidemia that disturbed redox balance resulting in the damage of polyunsaturated lipids in the mitochondrial membrane associated with a reduced export of $\mathrm{TG}$ and a subsequent accumulation in hepatocytes.

In the present study, ultrastructural examination of the hepatocytes of HFD-fed rats exhibited several drastic changes. Most of hepatocytes of HFD-fed rats appeared with abnormal nuclei, irregular nuclear envelope, degenerated mitochondria, dilated rough endoplasmic reticulum, large number of lysosomes, and rarified cytoplasm, in addition to accumulation of lipid droplets that increased in size forming large lipid vacuoles. Similarly, Abd Ellatif et al. ${ }^{[22]}$ and $\mathrm{Al}$ Humayed et $a .^{[23]}$ found similar ultrastructural observations and indicating that HFD induced NAFLD in rats causing steatosis that damaged the ultrastructure of the hepatocytes possibly via augmentation of lipid and oxidative stress. Ahishali et al. ${ }^{[24]}$ found similar ultrastructural features in nonalcoholic fatty liver in humans and reported that hepatosteatosis impaired oxidative phosphorylation and leading to increase the levels of ROS, inflammation, fibrosis, and mitochondrial abnormalities.

A significant increase in the activities of AST and ALT were recorded in the sera of animals fed on HFD comparing with the control group. These elevations may result from liver damage as supported by the histopathological and ultrastructural findings. Hassan et al. ${ }^{[19]}$ attributed that to an increase in the production of free radicals that initiate lipid peroxidation. Other studies reported the damage of parenchyma liver cells in different experimental animals fed on $\mathrm{HFD}^{[21,25]}$. Animals fed on HFD showed a significant increase in the concentrations of serum TC, TG, and LDL-C, while HDL-C concentration was significantly reduced, which may be due to lipid metabolic complications. Although the serum TG increased significantly at the $4^{\text {th }}$ week, it decreased significantly after 8 weeks, in HFD-fed animals revealing the effect of time of HFD feeding on serum TG level. These results were in agreement with Hussein et $a l^{[26]}$ who recorded a significant increase in serum levels of $\mathrm{TC}$, TG, very low density lipoprotein, and LDL, while the level of HDL was significantly decreased in obese female rats. In addition, Venkateshan et al. ${ }^{[27]}$ attributed that to the oxidative stress of HFD that highly correlated with a wide variety of inflammatory and metabolic disease states including obesity. Tveden-Nyborg et al. ${ }^{[21]}$ reported that HFD caused decrease in the concentration of $\mathrm{TG}$ due to its accumulation in hepatocytes rather than entering the systemic circulation. LDL is highly susceptible for oxidative damage and transforming into a harmful oxidized LDL, which was found to be elevated in plasma in patients with non-alcoholic steatohepatitis ${ }^{[28]}$. Under fat-rich conditions, LDL is taken up via the scavenger receptor-mediated pathway in an unlimited manner. In contrast to the LDL receptor, scavenger-receptor mediated uptake is not down-regulated in the presence of high concentration of LDL. Moreover, uncontrolled scavenger receptor-mediated uptake of LDL cholesterol into the liver is responsible for the formation of lipid droplets, foamy Kupffer cells, and cholesterol crystals, which both are crucial events for the development of nonalcoholic steatohepatitis ${ }^{[4]}$.

Recently, the use of herbal medicine has attracted significant interest among patients and researchers. The results of the present study showed no pathological changes were appeared in liver and serum of animals fed on cinnamon. ALGhwij et al. ${ }^{[29]}$ also reported that feeding on cinnamon alone did not change liver function or lipid profile in rats. Our results recorded a significant decrease in that body mass gained in HFDfed animals and this may be due to the reduction of mass of adipose tissue, which 
associated with the hypolipidemic effect of cinnamon. Other studies reported that cinnamon decrease the body weight in obese rats due to its polyphenols that have antiobesity activity through reducing the level of serum leptin hormone, which regulate the apatite $^{[6,30]}$

In the current study, the microscopically examination revealed a marked improvement in the histopathology and ultrastructure of liver of animals fed on HFD+cinnamon. These effects may be due to the antioxidant activity of cinnamon causing either inhibition or prevention of free radicals generation. Similarly, Niknezhad et al. ${ }^{[31]}$ found that cinnamon inhibited necrosis, infiltration of inflammatory cells, and decreased the fibrosis in liver, and attributed that to its ability in rebalancing antioxidant enzymes to eliminate cellular damage. The relief of the histopathological alterations in liver of obese rats was noticed after the treatment with cinnamon because of its eugenol, polyphenols, and flavanoids that reduced the level of oxidative stress, inflammatory, and apoptotic biomarkers, induced anti-obesity effect in rats fed on HFD, and inhibited the lipid peroxidation through ability to trap reactive carbonyl species ${ }^{[30,32,33]}$. Azab et al ${ }^{[34]}$ showed that eugenol ameliorated significantly the histopathological lesions of liver steatosis that induced by high fat high cholesterol diet. Similarly, El Ebiary and Khalaf $^{[35]}$ proved that cinnamon administration in concomitant with HFD ameliorated the hazardous effect of HFD on the structure of the liver of experimental rats, and attributed that to the antioxidant, anti-inflammatory, and lipolytic activity of cinnamon. Another clarification is that cinnamon may inhibit the hyperlipidemia through reducing the hepatic 5-hydroxy-3methylglutaryl-co-enzyme A (HMG-CoA) reductase activity (a rate limiting enzyme involved in regulating cholesterol metabolism or biosynthesis and decrease serum TC level); therefore, it acts as a hypocholesterolemic agent ${ }^{[36]}$. Moreover, Lee et $a .^{[37]}$ found that cinnamate, a phenolic compound present in cinnamon bark, is accountable for its protective action; and the activation of antioxidant enzymes due to its redox effects is responsible for its free-radical scavenging activity, transitionmetal-chelating activity, and singlet-oxygen quenching capacity.

In the present study, the activity of serum transaminases (AST and ALT) have been decreased by adding cinnamon to HFD when compared with animals fed on HFD only, indicating the hepatoprotective effect of cinnamon. Similar results were recorded by Kassaee et $a .^{[38]}$ who suggested that cinnamon may play an important role in improving the liver function and have protective effect against the hepatocellular injuries. Similarly, ALGhwij et $a l^{[29]}$ reported that cinnamon was completely improved the AST and ALT activities in serum of animals treated with trichloroethylene. Askari et al. ${ }^{[14]}$ reported that cinnamon supplementation could reduce the main characteristics of NAFLD by modification of insulin resistance, liver enzymes, and the inflammatory markers. Moreover, Roussel et al. ${ }^{[39]}$ also attributed that to the antioxidant activity of cinnamon.

Concerning the lipid profile parameters, our results indicated that adding cinnamon to HFD significantly alleviated the dyslipidemia of HFD-fed animals. Abeysekera et $a{ }^{[40]}$ established that the bark extracts of Ceylon cinnamon might have a direct role in lipid metabolism by reducing TC and TG concentrations, and lowering the free fatty acids by its strong lipolytic activity, due to the presence of cinnamate that inhibits the hepatic HMG-CoA reductase activity. These results correlate with the findings of Iqbal et al. ${ }^{[41]}$ who found that cinnamon powder improved the serum lipid profile parameters in albino rats, which may be proposed a direct role in the lipid metabolism. The cinnamon polyphenols inhibit the formation of hyperlipidemic fatty livers in mice through decreasing the hepatic lipid accumulation, sterol regulatory element, and fatty acid synthase expression, and increasing the 
lipoprotein lipase, and cholesterol $7 \alpha$ hydroxylase expression ${ }^{[42]}$. The dietary fiber that found at a relatively high level in cinnamon can improve the lipid profile by decreasing the lipid absorption in the intestine, as well as it contains antioxidant vitamins that can enhance lipid catabolism ${ }^{[38]}$. In addition, cinnamon decrease the level of LDL by increasing LDL-receptors in the liver that involved in the scavenging of LDL; thus the LDL-receptor gene expression is another probable mechanism of hypocholestrolic effect of cinnamon ${ }^{[38]}$. Maierean et al. ${ }^{[43]}$ suggested that cinnamon decreased the absorption of chylomicron, the small fat globules that serve to transport fat from its port of entry in the intestine to the liver and to adipose tissue, and potentially increased the TG uptake by adipocytes.

In conclusion, the accumulation of fats in liver of obese rats plays a critical role in the generated histological and ultrastructure alterations that could be alleviated by cinnamon supplementation due to its ability to provide antidyslipidemic effect and protect from liver injury.

\section{FINANCIAL SUPPORT}

This research received no specific grant from any funding agency in the public, commercial or not-for-profit sectors.

\section{CONFLICT OF INTEREST}

The authors have no potential financial conflict of interest

\section{REFERENCES}

[1] Wang, J.; He, K.; Yang, C. et al. (2019). Dietary cholesterol is highly associated with severity of hyperlipidemia and atherosclerotic lesions in heterozygous LDLRdeficient hamsters. Int $\mathrm{J}$ Mol Sci, 20(14): E3515 (doi: 10.3390/ijms 20143515).

[2] Subramanian, S.; Goodspeed, L.; Wang, S. et al. (2011). Dietary cholesterol exacerbates hepatic steatosis and inflammation in obese LDL receptor-deficient mice. J Lipid Res, 52(9): 1626-1635.

[3] Delbosc, S.; Paizanis, E.; Magous, R. et al. (2005). Involvement of oxidative stress and NADPH oxidase activation in the development of cardiovascular complications in a model of insulin resistance, the fructose-fed rat. Atherosclerosis, 179: 43-49.

[4] Walenbergh, S. M. and Shiri-Sverdlov, R. (2015). Cholesterol is a significant risk factor for non-alcoholic steatohepatitis. Expert Rev Gastroenterol Hepatol, 9(11): 1343-1346.

[5] Kawatra, P. and Rajagopalan, R. (2015): Cinnamon: mystic powers of a minute ingredient. Pharmacognosy Res, 7(1): S1-6.

[6] Mousavi, S. M.; Rahmani, J.; KordVarkaneh, H. et al. (2020). Cinnamon supplementation positively affects obesity: a systematic review and doseresponse meta-analysis of randomized controlled trials. Clin Nutr, 93: 123133.

[7] Abu Bakar; Yao, P.-C.; Ningrum, $\mathrm{V}$. et al. (2020). Beneficial biological activities of Cinnamomum osmophloeum and its potential use in the alleviation of oral mucositis: a systematic review. Biomedicines, 8: 3 (DOI:10.3390/ biomedicines8010003).

[8] Hamidpour, R.; Hamidpour, M.; Hamidpour, S. et al. (2015). Cinnamon from the selection of traditional applications to its novel effects on the inhibition of angiogenesis in cancer cells and prevention of Alzheimer's disease, and a series of functions such as antioxidant, anticholesterol, antidiabetes, antibacterial, antifungal, nematicidal, acaracidal, and repellent activities. $\mathbf{J}$ Tradit Complement Med, 5(2): 66-70.

[9] Borzoei, A.; Rafraf, M.; Niromanesh, S. et al. (2018). Effects of cinnamon supplementation on antioxidant status 
and serum lipids in women with polycystic ovary syndrome. J Tradit Complement Med, 8: 128-133.

[10] Chan, K. W.; Khong, N. M. H.; Iqbal, S. et al. (2014). Cinnamon bark deodorised aqueous extract as potential natural antioxidant in meat emulsion system: a comparative study with synthetic and natural food antioxidants. J Food Sci Technol, 51(11): 3269-3276.

[11] Jakhetia, V.; Patel, R.; Khatri, P. et al. (2010). Cinnamon: a pharmacological review. J Adv Sci Res, 1(2): 19-23.

[12] Tuzcu, Z.; Orhan, C.; Sahin, N. et al. (2017). Cinnamon polyphenol extract inhibits hyperlipidemia and inflammation by modulation of transcription factors in high-fat dietfed rats. Oxid Med Cell Longev, 2017: 1583098 (DOI: 10.1155/2017/ 1583098).

[13] Gunawardena, D.; Karunaweera, N.; Lee, S. et al. (2015). Antiinflammatory activity of cinnamon (C. zeylanicum and C. cassia) extracts - identification of E-cinnamaldehyde and $o$-methoxy cinnamaldehyde as the most potent bioactive compounds. Food Funct, 6(3): 910-919.

[14] Askari, F.; Rashidkhani, B. and Hekmatdoost, A. (2014). Cinnamon may have therapeutic benefits on lipid profile, liver enzymes, insulin resistance, and high-sensitivity $\mathrm{C}$ reactive protein in nonalcoholic fatty liver disease patients. Nutr Res, 34(2): 143-148.

[15] Rahman, S.; Begum, H.; Rahman, Z. et al. (2013). Effect of cinnamon (Cinnamomum cassia) as a lipid lowering agent on hypercholesterolemic rats. Journal of Enam Medical College, 3(2): 94-98.

[16] Xia, Z. H.; Chen, W. B.; Shi, L. et al. (2020). The underlying mechanisms of curcumin inhibition of hyperglycemia and hyperlipidemia in rats fed a highfat diet combined with STZ treatment.
Molecules, 25(2): E271 (DOI:10.3390/ molecules25020271).

[17] Fidèle, N.; Joseph, B.; Emmanuel, T. et al. (2017). Hypolipidemic, antioxidant and anti-atherosclerogenic effect of aqueous extract leaves of Cassia occidentalis Linn (Caesalpiniaceae) in diet-induced hypercholesterolemic rats. BMC Complement Altern Med, 17:76 (DOI 10.1186/s12906-017-1566-x).

[18] Jo, J.; Gavrilova, O.; Pack, S. et al. (2009). Hypertrophy and/or hyperplasia: dynamics of adipose tissue growth. PLoS Comput Biol, 5(3): e1000324 (DOI: 10.1371/journal.pcbi. 1000324).

[19] Hassan, N. F.; Soliman, G. M.; Okasha, E. F. et al. (2018). Histological, immunohistochemical, and biochemical study of experimentally induced fatty liver in adult male albino rat and the possible protective role of pomegranate. $\mathrm{J}$ Microsc Ultrastruct, 6(1): 44-55.

[20] Aborhyem, S.; Ismail, H.; Agamy, N. et al. (2016). Effect of Moringa oleifera on lipid profile in rats. Journal of High Institute of Public Health, 46: 8-14.

[21] Tveden-Nyborg, P.; Birck, M. M.; Ipsen, D. H. et al. (2016). Dietinduced dyslipidemia leads to nonalcoholic fatty liver disease and oxidative stress in guinea pigs. Transa Res, 168: 146-160.

[22] Abd Ellatif, M.; El Karib, A. O.; Dallak, M. et al. (2018). Vitamin E protects against hepatocyte ultrastructural damage induced by high fat diet in a rat model of pre-diabetes. Int J Morphol, 36(4): 1350-1355.

[23] Al Humayed, S.; Eid, R. A.; Shatoor, A. S. et al. (2017). Differential therapeutic effects of Crataegus aronia and simvastatin on the hepatocyte ultrastructure in hepatic steatosis. Int J Morphol, 35(2): 578-583. 
[24] Ahishali, E.; Demir, K.; Ahishali, B. et al. (2010). Electron microscopic findings in non-alcoholic fatty liver disease: is there a difference between hepatosteatosis and steatohepatitis? J Gastroenterol Hepatol, 25(3): 619-626.

[25] Elshazly, S. M. (2015): Ameliorative effect of nicorandil on high fat diet induced non-alcoholic fatty liver disease in rats. Eur J Pharmacol, 748(5): 123-132.

[26] Hussein, S. A.; Abdel-Magid, A. D. and Fareed, F. A. (2017). Biochemical effect of resveratrol on lipids profile and hepatic oxidative stress in experimentally induced obesity in female rats. BVMJ, 32(1): 67-74.

[27] Venkateshan, S.; Subramaniyan, V.; Chinnasamy, V. et al. (2016). Anti-oxidant and anti-hyperlipidemic activity of Hemidesmus indicus in rats fed with high-fat diet. Avicenna J Phytomed; 6(5): 516-525.

[28] Chalasani, N.; Deeg, M. A. and Crabb, D. W. (2004). Systemic levels of lipid peroxidation and its metabolic and dietary correlates in patients with nonalcoholic steatohepatitis. Am J Gastroenterol, 99(8):1497-1502.

[29] ALGhwij, L. M.; Ghoneim, A. M.; Said, A. E. et al. (2012). Effect of some herbal plants on liver function of rats treated with trichloroethylene. The Egyptian Journal of Hospital Medicine, 46:7-16.

[30] Abd El-Rahman, G. I. (2018). Evaluation the efficacy of combined mixture of spirulina platensis and cinnamon extracts in overweight rats fed on a fatty diet. Life Sci J, 15(7): $37-46$.

[31] Niknezhad, F.; Sayad-Fathi, S.; Karimzadeh, A. et al. (2019). Improvement in histology, enzymatic activity, and redox state of the liver following administration of Cinnamomum zeylanicum bark oil in rats with established hepatotoxicity. Anat Cell Biol, 52(3): 302-311.
[32] Ismail, N. S. (2014). Protective effects of aqueous extracts of cinnamon and ginger herbs against obesity and diabetes in obese diabetic rat. World Journal of Dairy and Food Sciences, 9(2): 145-153.

[33] Peng, X.; Cheng, K. W. and Ma J. (2008). Cinnamon bark proanthocyanidins as reactive carbonyl scavengers to prevent the formation of advanced glycation endproducts. J Agric Food Chem, 56(6):19071911.

[34] Azab, M. H. F.; Zaki, A. E.; Abdel Azim, N. M. S. et al. (2017). Eugenol ameliorates pathogenic features in experimental model of NAFLD in rats. Az J Pharm Sci, 56(2): 115-122.

[35] El Ebiary, F. H. and Khalaf, G. (2014). The effect of high fructose diet on the structure of liver of albino rat and the possible protective role of cinnamon. Light and electron microscopic study. IOSR-JDMS, 13(6): 46-53.

[36] Kannappan, S.; Jayaraman, T.; Rajasekar, P. et al. (2006). Cinnamon bark extract improves glucose metabolism and lipid profile in the fructose-fed rat. Singapore Med J, 47(10): 858-863.

[37] Lee, J. S.; Jeon S. M.; Park, E. M. et al. (2003). Cinnamate supplementation enhances hepatic lipid metabolism and antioxidant defense systems in high cholesterolfed rats. J Med Food, 6(3):183-191.

[38] Kassaee, S. M.; Goodarzi, M. T.; Hayati Roodbari, N. et al. (2017). The effects of Cinnamomum zeylanicum on lipid profiles and histology via up-regulation of LDL receptor gene expression in hamsters fed a high cholesterol diet. Jundishapur J Nat Pharm Prod, 12(3): e37340 (DOI: 10.5812/jjnpp.373403).

[39] Roussel, A. M.; Hininger, I.; Benaraba, R. et al. (2009). Antioxidant effects of a cinnamon extract in people with impaired fasting glucose that 
are overweight or obese. J Am Coll Nutr, 28:16-21.

[40] Abeysekera, W. P. K. M.; Arachchige, S. P. G. and Ratnasooriya, W. D. (2017). Bark extracts of Ceylon cinnamon possess antilipidemic activities and bind bile acids in vitro. Evid Based Complement Alternat Med, 2017: 7347219 (DOI: 10.1155/2017/7347219).

[41] Iqbal, Z.; Ashraf, T.; Khan, A. A. et al. (2015). Antihyperlipidemic efficacy of cinnamon in albino rats. Asian J Agri Biol, 4: 8-16.
[42] Cui, Y.; Wang, X.; Xue, J. et al. (2014). Chrysanthemum morifolium extract attenuates highfat milk-induced fatty liver through peroxisome proliferatoractivated receptor $\alpha$-mediated mechanism in mice. Nutr Res, 34(3): 268-275.

[43] Maierean, S. M.; Serban, M. C.; Sahebkar, A. et al. (2017). The effects of cinnamon supplementation on blood lipid concentrations: a systematic review and meta-analysis. J Clin Lipidol, 11(6): 1393-1406.

\section{How to cite this article:}

Arisha, S. M.; Saker, S. A. and Abd El-Haseeb, F. R. (2020). Cinnamon reduces dyslipidemia and liver steatosis induced by high fat diet in albino rats: histological, ultrastructural, and biochemical studies. Egyptian Journal of Zoology, 73: 67-83 (DOI: 10.12816/ejz.2020.29834.1031). 
نبات القرفة يقلل من فرط شحوم الام وتثمع الكبد المُحدثان بالوجبة الغذائية عالية الدسم

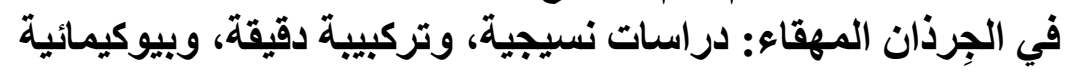

سماح محمد عريثه، صابر عبدالرحمن صقر، فاطمة رجب عبد الحسيب

قسم علم الحيو ان، كلية العلوم، جامعة المنوفية، المنوفية، جمهورية مصر العربية

تسبب الوجبات الغذائية عالية الدسم فرط شحمبات الدم ومرض الكبد الدهني غير الكحولي. ولنبات القرفة العديد من التأثيرات العلاجية كمضاد للإلتهابات ومضاد التان للأكسدة الخلوية.

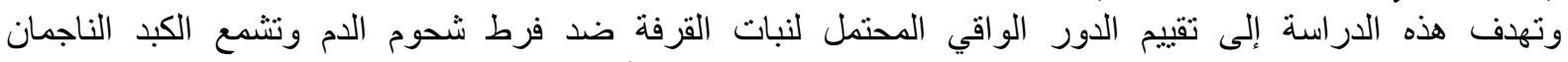

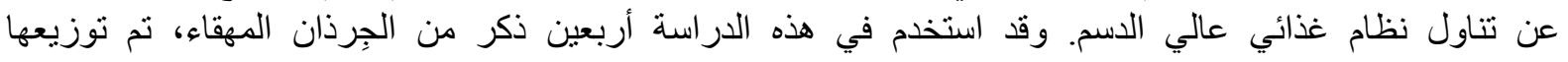

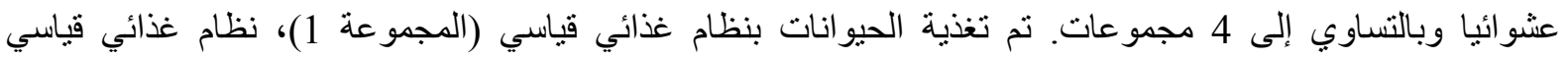

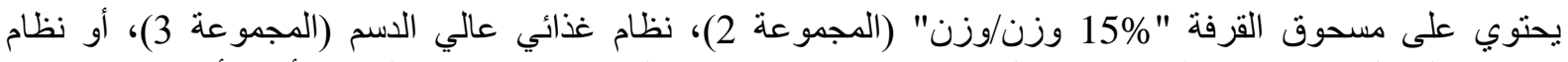

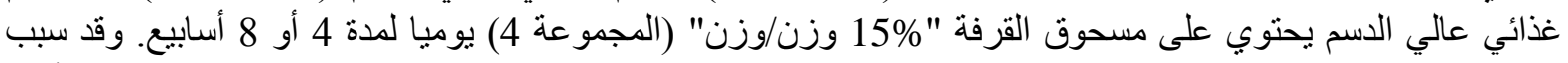

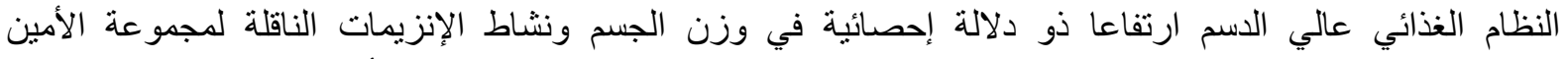

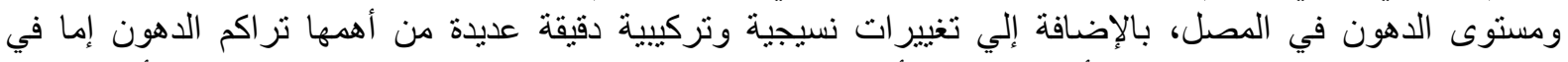

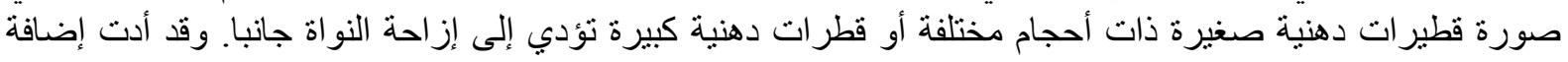

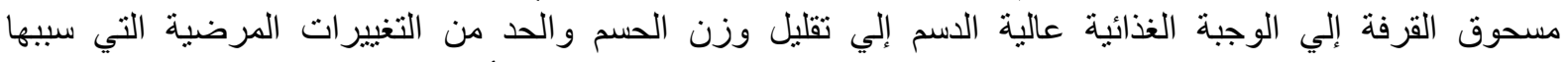

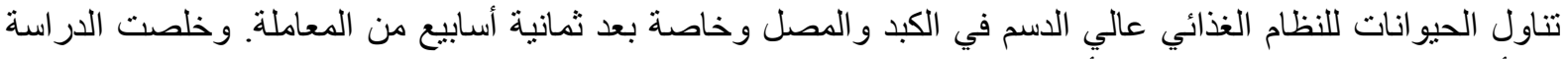

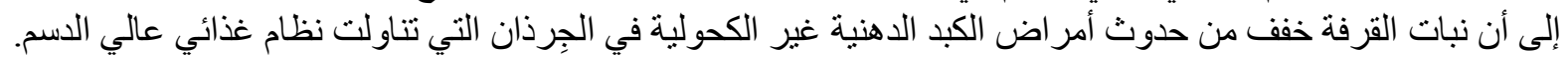

